\title{
Measurement of the Longitudinal Wakefield and the Bunch Shape in the SLAC Linac*
}

\author{
K.L.F. Bane, F.-J. Decker, J.T. Seeman, F. Zimmermann \\ Stanford Linear Accelerator Center, Stanford University, Stanford, CA 94309
}

\begin{abstract}
We report on measurements of the bunch energy spectrum at the end of the SLAC linac. Using the spectra obtained for two different linac rf phases we obtain both the bunch induced voltage and the longitudinal distribution of the bunch. The measurement results are compared with theoretical predictions. In particular, the induced voltage is in good agreement with that obtained using the calculated wake function for the SLAC linac. This measurement technique may be useful for monitoring changes of the linac bunch shape in the SLC.
\end{abstract}

DISTARUTION OF THIS DOCUMENT IS UNLIMITED

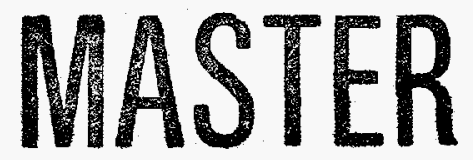

Presented at 1997 Particle Accelerator Conference

Vancouver, British Columbia, Canada

May 12-16, 1997

${ }^{*}$ Work supported by Department of Energy contract DE-AC03-76SF00515. 


\title{
Measurement of the Longitudinal Wakefield and the Bunch Shape in the SLAC Linac*
}

\author{
K.L.F. Bane, F.-J. Decker, J.T. Seeman, F. Zimmermann \\ Stanford Linear Accelerator Center, Stanford University, CA 94309, USA
}

\section{Abstract}

We report on measurements of the bunch energy spectrum at the end of the SLAC linac. Using the spectra obtained for two different linac if phases we obtain both the bunch induced voltage and the longitudinal distribution of the bunch. The measurement results are compared with theoretical predictions. In particular, the induced voltage is in good agreement with that obtained using the calculated wake function for the SLAC linac. This measurement technique may be useful for monitoring changes of the linac bunch shape in the SLC.

\section{INTRODUCTION}

In a linear accelerator the final energy spectrum of a bunch depends on the applied rf voltage, the bunch length, and the wakefield. If the beam phase is chosen sufficiently far off crest so that (1) there exists a 1-to-1 correspondence between the longitudinal position within the bunch and the final energy, and (2) the wakefield effect can be ignored, then a single measurement of the beam energy spectrum at the end of the linac suffices to reconstruct the bunch shape. Normally there are practical limitations as to how far off crest one can go and it may be the case that condition (1) can be satisfied but condition (2) cannot. In such a case a single measurement of the energy spectrum does not suffice for obtaining the bunch shape. However, if the energy spectrum is measured for at least two different beam phases, both of which are far enough off crest to satisfy condition (1), then both the bunch shape and the bunch wake (the induced voltage) can be reconstructed[1].

Estimating the bunch length from spectrum measurements in the SLAC linac has been done before[2, 3]; in both cases, however, wakefield effects were ignored. In addition, the parasitic mode losses-the average of the induced voltage-have been measured as a function of current [3]. In this report, we describe the first measurement of both the induced voltage and the bunch shape in the SLAC linac, using the technique mentioned above. This method is straightforward, and possibly a simpler alternative to bunch length measurements using a streak camera. A quick measurement of the electron or positron bunch shapes in the SLAC linac can be of great help in adjusting the rf parameters of the bunch compressors, which are located between the damping rings and the linac, so as to optimize the luminosity at the interaction point of the Stanford Linear Collider (SLC) [4].

\footnotetext{
* Work supported by the U.S. Department of Energy under contract DE-AC03-76SF00515.
}

\section{THEORY[1]}

Consider a bunch of charged particles that pass through the linac. The peak energy gain of the $\mathrm{rf}$ in the linac is $E_{a}$; the bunch phase with respect to the crest is $\phi$, with a more negative value of phase a position more forward on the $\mathrm{rf}$ wave. Let us assume that $E_{f} / E_{0} \gg 1$, with $E_{f}$ and $E_{0}$, respectively, the final and the initial energy of the beam, so that we can ignore the component of energy variation that is uncorrelated with longitudinal position. Then the relative energy of a particle at position $z$ within the bunch becomes

$$
\delta(z)=\left[E_{0}+E_{\mathrm{a}} \cos \left(k_{r f} z+\phi\right)+e V_{\text {ind }}(z)\right] / E_{f}-1,
$$

with $k_{r f}$ the rf wave number and $V_{i n d}(z)$ the induced voltage, given by

$$
V_{\text {ind }}(z)=-e N \int_{0}^{\infty} W_{z}\left(z^{\prime}\right) \lambda_{z}\left(z-z^{\prime}\right) d z^{\prime},
$$

with $N$ the bunch population, $W_{z}$ the Green function wakefield, and $\lambda_{z}$ the normalized longitudinal charge distribution.

By knowing both $\lambda_{z}(z)$ and $\delta(z)$ over the bunch length we can compute the energy distribution $\lambda_{\delta}(\delta)$. Conversely, if we know $\lambda_{\delta}(\delta)$ and $\delta(z)$, we can calculate $\lambda_{z}(z)$, provided $\delta(z)$ is monotonic over the bunch. Let us assume that this is the case. Then

$$
\lambda_{z}(z)=\lambda_{\delta}(\delta(z))\left|\delta^{\prime}(z)\right|
$$

Suppose now that we know $E_{0}, E_{a}, E_{f}, k_{r f}$, and $\phi$. Without knowing the induced voltage we cannot, in general, obtain $\lambda_{z}$ from $\lambda_{\delta}$, since $\delta$ depends also on $V_{i n d}$. Only if $e V_{i n d}^{\prime}$ is small compared to $\delta^{\prime} E_{f}$ over the bunch does a single measurement of $\lambda_{\delta}$ suffice to give $\lambda_{z}$.

Suppose we measure the bunch spectrum twice: with the beam at phase $\phi^{a}$ we obtain $\lambda_{\delta}^{a}$, and then with the beam at $\phi^{b}$ we obtain $\lambda_{\delta}^{b}$. We assume the phases are chosen so that $\delta(z)$ is monotonic for both measurements. For the first measurement Eq. (3) becomes

$$
\lambda_{z}(z)=\lambda_{\delta}^{a}\left|E_{a} k_{r f} \sin \left(k z+\phi^{a}\right)-e V_{i n d}^{\prime}(z)\right| / E_{f},
$$

and a similar equation, with superscript $b$ replacing superscript $a$, holds for the second measurement. Combining these two equations we obtain

$$
V_{i n d}^{\prime}(z)=\frac{E_{a} k_{r f}}{\lambda_{\delta}^{a} \pm \lambda_{\delta}^{b}}\left[\lambda_{\delta}^{a} \sin \left(k z+\phi^{a}\right) \pm \lambda_{\delta}^{b} \sin \left(k z+\phi^{b}\right)\right] \text {. }
$$

In Eq. (5) (and below) the upper symbol of \pm applies if the sign of $\delta^{\prime}(z)$ is different for the two measurements, otherwise the lower symbol applies. The right hand side of 
Eq. (5) is a function both of $z$ and-through the argument of $\lambda_{\delta}$-of $V_{\text {ind }}(z)$. Eq. (5) is therefore a first order nonlinear differential equation which we can solve numerically for the unknown $V_{\text {ind }}(z)$. As initial condition we take $V_{\text {ind }}$ at the front of the bunch to be zero. Once $V_{i n d}$ is known we obtain $\lambda_{z}$ using

$$
\lambda_{z}(z)=\frac{E_{a} k_{r f} \lambda_{\delta}^{a} \lambda_{\delta}^{b}}{E_{f}\left|\lambda_{\delta}^{a} \pm \lambda_{\delta}^{b}\right|}\left|\sin \left(k z+\phi^{a}\right)-\sin \left(k z+\phi^{b}\right)\right| .
$$

\section{ENERGY SPECTRA}

The measurements were performed with the machine in a Final Focus Test Beam (FFTB) configuration. The number of particles per bunch was relatively small $N=7.5 \times 10^{9}$; $E_{0}=1.19 \mathrm{GeV}, E_{f}=46 \mathrm{GeV}, k_{r f}=60 \mathrm{~m}^{-1}$. The peak rf voltage of the damping ring was $800 \mathrm{kV}$. The bunch compressor voltage was set to $V_{c}=32.4 \mathrm{MV}$. (However, there was circumstantial evidence that the actual compressor voltage was substantially lower, a suspicion, as we shall see, that is supported by our analysis.) For these conditions, the expected rms bunch length in the linac was about $0.5 \mathrm{~mm}$. The FFTB final focus lattice was modified to give a large dispersion $(\eta=60 \mathrm{~cm})$ and a small beta function (corresponding to a beam size of $150 \mu \mathrm{m}$ ) at the wire monitor in the FFTB dump line that was used for the measurements. The wire size is $75 \mu \mathrm{m}$, corresponding to an energy

- resolution of about $0.01 \%$.

Our first measurement was to check that the calibration of the phase shifter was correct, and to find the phase corresponding to the top of the rf crest (which we designate as $\phi=0$ ). For this measurement a beam-position monitor (BPM) at a high dispersion point was used to record the average beam energy as the phase knob was varied. The results are shown in Fig. 1, with the phase shifted to fit the expected dependence (the curve). The deviation seen for phases below $-5^{\circ}$ is likely due to spraying of the BPM by beam particles.

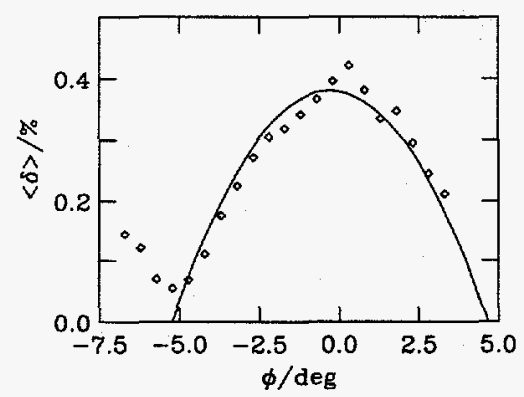

Figure 1: A'calibration measurement.

We then performed a series of energy spectrum measurements using the wire monitor, for different linac phases, first without and then with the beam energy feedback activated. When turned on, this feedback tries to maintain a constant average beam energy in the FFTB line without changing $\phi$. We were not successful in obtaining good data for $\phi<-6.7^{\circ}$ or $\phi>2.3^{\circ}$. Figure 2 displays 6 representative spectra, all of which were measured with the energy feedback on. For comparison, Fig. 3 presents two simulated spectra, with phases corresponding to those of Fig. 2a and $\mathrm{f}$, but with $V_{c}$ reduced to $30.4 \mathrm{MV}$. The agreement with the measurements is quite good. In the bottom frames of Fig. 3 contours of phase space are also shown.

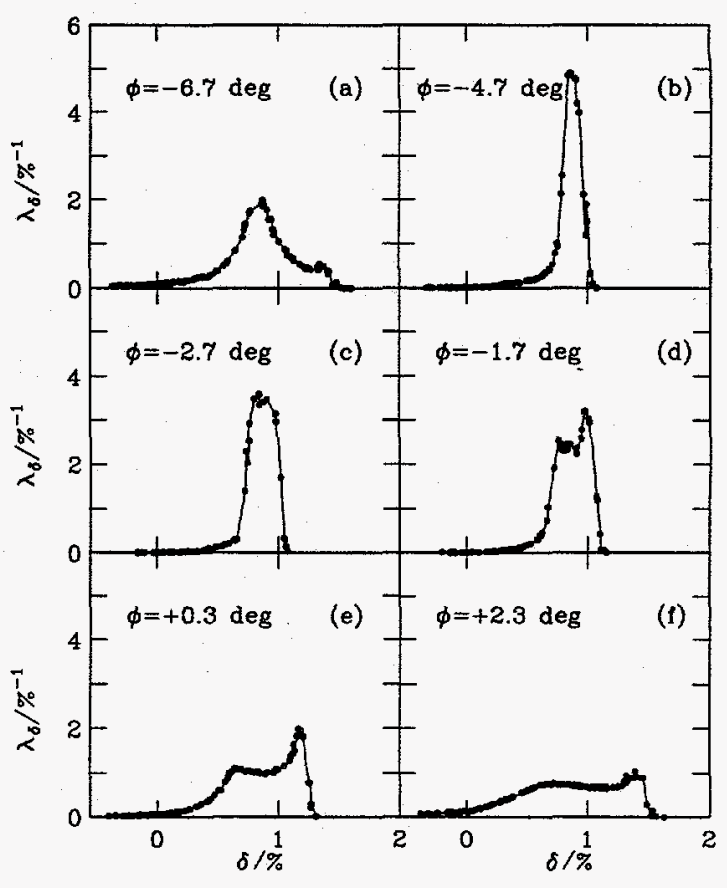

Figure 2: Six representative measurements of the energy spectrum. For all cases shown the energy feedback is on.
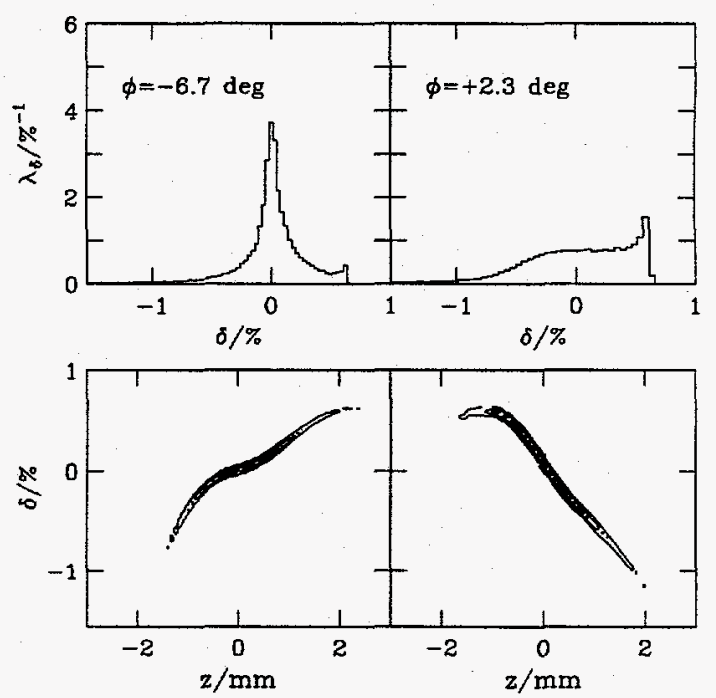

Figure 3: Two simulated spectra and the corresponding contours in phase space, assuming $V_{c}=30.4 \mathrm{MV}$.

The spectral measurements are summarized in Fig. 4. In Fig. $4 \mathrm{a}$ the area under the spectrum curve is plotted; we see that it deviates by no more than $10 \%$. Figure $4 \mathrm{~b}$ shows the centroid energy. The curve gives the expected behavior (up 
to an arbitrary amount of shift) when the feedback is off. With feedback on $\langle\delta\rangle$ remains fairly constant. With feedback off, however, the data points do not exactly follow the expected behavior, which may be due to klystrons cycling on or off. In Figs. 4c-d we plot the rms and the FWHM width of the spectra. We note that the minimum in both plots occurs when the beam is $4-5^{\circ}$ in front of the rf crest. The curves in Figs. $4 c-d$ give results obtained from simulations, for the nominal compressor setting $V_{c}=32.4 \mathrm{MV}$ (solid), and for $V_{c}=30.4 \mathrm{MV}$ (dashes). The latter curves agree much better to the measurements, adding support to the belief that the compressor voltage was low.
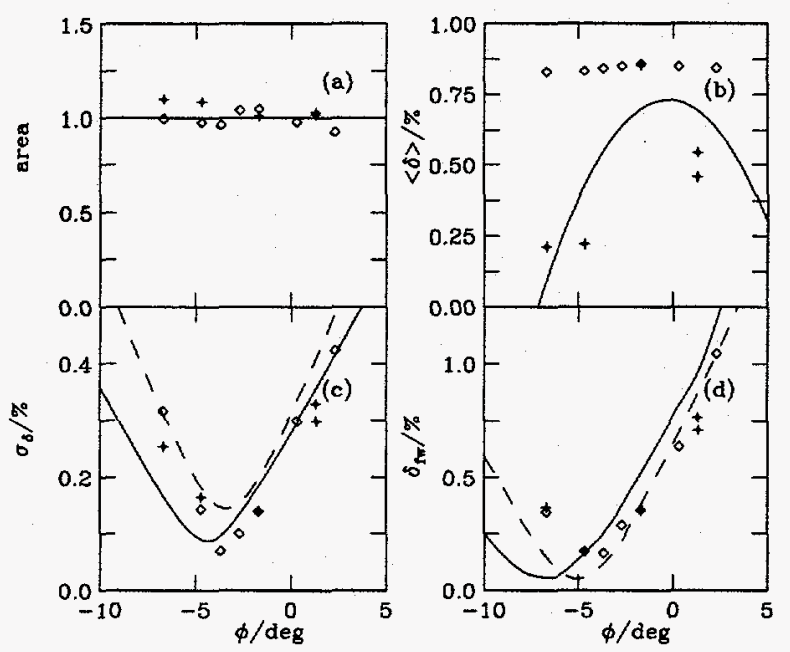

Figure 4: The area (a), the centroid position $\langle\delta\rangle(\mathrm{b})$, the rms length $\sigma_{\delta}$ (c), and the FWHM $\delta_{f w}$ (d) of the measured spectra as functions of phase $\phi$. The diamonds signify measurements with energy feedback, the crosses, without. The curves are described in the text.

\section{BUNCH SHAPE AND WAKEFIELD}

First, as an example, let us neglect the effect of the wakefield (let $V_{\text {ind }}(z)=0$ ) and invert Eq. 4 using the spectrum of Fig. 2a. Figure 5 shows the result. The FWHM width $z_{f w}=0.53 \mathrm{~mm}$, which is smaller than is theoretically possible. This demonstrates that even at this low current the wakefields can be important for this measurement.

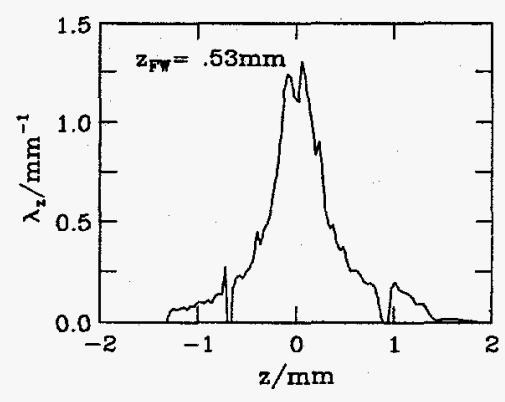

Figure 5: Result of the inversion using the spectrum of Fig. 2a when the effect of the wakefield is neglected.
For our two-phase calculations we would like the phases to be at opposite sides of $\phi=0$ [otherwise the terms in the denominators of Eqs. (5),(6) subtract], and as far apart as possible. We choose the spectra shown in Fig. $2 \mathrm{a}$ and $\mathrm{f}$. We see from the contour plots of Fig. 3 that for the $\phi=2.3^{\circ}$ case we expect that $\delta(z)$ is not monotonic at the head of the bunch, for $z<-1 \mathrm{~mm}$. So we begin our solution at $z=-1 \mathrm{~mm}$ at which point we set $V_{i n d}=-40 \mathrm{MV}$. The results are shown in Fig. 6.

Shown in Fig. 6a is the bunch shape; the gaussian fit (the dashes) has $z_{f w}=1.50 \mathrm{~mm}$, which is similar to our expectations when $V_{c}=30.4 \mathrm{MV}$ (at $V_{c}=32.4 \mathrm{MV}$ we expect $z_{f w}=1.2 \mathrm{~mm}$ ). Note that the area under the curve equals 0.82 , and not 1 , probably due to the nonmonotonicity problem discussed above. In Fig. $6 \mathrm{~b}$ we show $V_{\text {ind }}$; it is smooth because it is the result of an integration. As a check we convolve the gaussian fit bunch shape with the calculated SLAC wake function[5] according to Eq. (2) and obtain the dashed curve. The agreement over the core of the bunch is good. Finally, we should point out that although the details may change, the basic features of our results do not seem to be very sensitive to exactly how we begin the calculation at the head of the bunch.
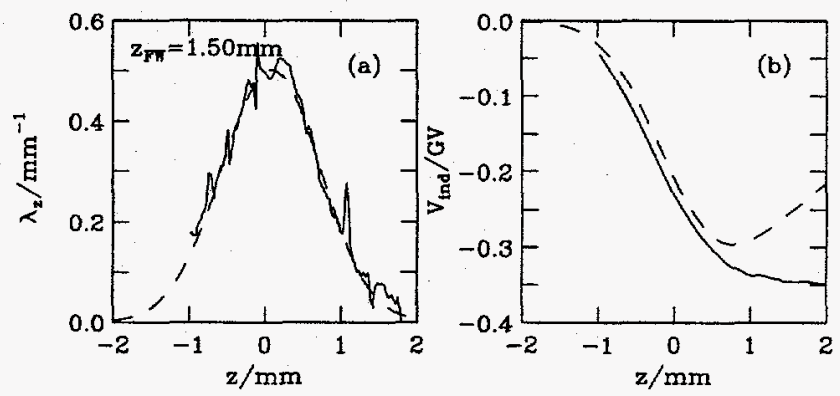

Figure 6: Result of the inversion using the spectra of Figs. 2a and f: (a) the bunch shape and (b) the measured induced voltage (the solid curves). The dashed curves are discussed in the text.

\section{ACKNOWLEDGEMENTS}

The authors thank P. Tenenbaum for setting up the FFTB optics for this measurement, and K.B. thanks R. Warnock for a helpful discussion on this topic many years ago.

\section{REFERENCES}

[1] K.L.F. Bane, SLAC/AP-80 (1990).

[2] J. Seeman, Proc. of the 1988 Linear Acc. Conf., CEBAF, 1988, p. 487.

[3] K. Bane et al., Proc. of the $2^{\text {nd }}$ European Particle Acc. Conf., Nice, France, 1990, p. 1762.

[4] K.L.F. Bane, P. Chen, F. Zimmermann, "Beam-beam simulations with non-Gaussian distributions for SLC and SLC2000 ", these proceedings (1997).

[5] K. Bane and P. Wilson, Proc. of the $11^{\text {th }}$ Int. Conf. on HighEnergy Acc., CERN, 1980, p. 592. 


\section{DISCLAIMER}

This report was prepared as an account of work sponsored by an agency of the United States Government. Neither the United States Government nor any agency thereof, nor any of their employees, makes any warranty, express or implied, or assumes any legal liability or responsibility for the accuracy, completeness, or usefulness of any information, apparatus, product, or process disclosed, or represents that its use would not infringe privately owned rights. Reference herein to any specific commercial product, process, or service by trade name, trademark, manufacturer, or otherwise does not necessarily constitute or imply its endorsement, recommendation, or favoring by the United States Government or any agency thereof. The views and opinions of authors expressed herein do not necessarily state or reflect those of the United States Government or any agency thereof. 


\section{DISCLAMMER}

Portions of this document may be illegible in electronic image products. Images are produced from the best available original document. 